\title{
狳学通极
}

\section{$\mathbf{C u}-\mathbf{Z n}-\mathbf{A l}$ 合金贝氏体的层错形成研究*}

\author{
李春明(1) (2) 方鸿生 ${ }^{(1)}$ 郑燕康 ${ }^{(1)}$ 王家军 ${ }^{(1)}$ 杨志刚 ${ }^{(1)}$
}

(1) 清华大学材料科学与工程系, (2) 清华大学现代应用物理系, 北京 100084)

\section{关键词 Cu-Zn-Al 合金 贝氏体 层错}

贝氏体相变是固态相变的主要类型之一, 关于它的形成理论代表了一种片状相的形成理 论, 这种相变涉及钢铁、有色合金及陶瓷等材料领域的有关片状相的形成, 具有重大的理论和 实际意义. 但是, 由于贝氏体相变本身的复杂性, 尽管材料科学工作者对其进行了半个多世 纪的研究, 至今仍有许多问题悬而未决, 扩散和切变两大学派根据各自的实验结果, 观点对 立, 分歧严重.

Wayman $^{[1]}$, Christian $^{[2]}$ 和 Lieberman ${ }^{[3]}$ 等认为, 切变相变的产物内部必然会存在诸如孪 晶、堆垛层错或能产生点阵不变形变 (LID) 的位错等缺陷. 具体对 $\mathrm{Cu}-\mathrm{Zn}-\mathrm{Al}$ 合金贝氏体而 言, 这种缺陷就是其内部层错.

现已知道, $\mathrm{Cu}-\mathrm{Zn}-\mathrm{Al}$ 合金贝氏体的亚结构为层错 ${ }^{44,9}$, 但贝氏体是否一旦形成即有层错存在 则是一个争议很大的理论问题. 切变观点认为 ${ }^{[6-8]}$, 贝氏体是不变平面应变特征的马氏体型相 变产物, 层错是作为协调切变应力的缺陷而伴随贝氏体的产生出现的. 换言之, 贝氏体一旦 形成即有层错存在. 然而, 不少实验结果表明 ${ }^{[9,10]}$, 贝氏体在形成初期内部并无层错存在.

我们进一步发现 ${ }^{[1]}, \mathrm{Cu}-\mathrm{Zn}-\mathrm{Al}$ 合金贝氏体的生长明显分为 3 个阶段: 初生态、中间态及退 化态. 初生态贝氏体不含内部层错, 并且其宽面和生长端面上均有台阶存在 ${ }^{[12]}$. 中间态贝氏 体内有层错存在, 而退化态贝氏体内的层错则逐渐消失, 最终转变为平衡相. 目前, 切变和扩 散学派对中间态和退化态贝氏体的结构特征并无异议, 其分歧在于贝氏体的初生态是否含有 层错, 因为这是关系到贝氏体是否由切变形成的重要问题.

显而易见, 根据我们的观察结果, 即贝氏体的初生态无层错而中间态有层错, 则在这两者 之间的某一阶段必定会出现层错形成的过程, 但迄今为止,这还只是一种合理的推测, 国内外 文献尚未报道有关层错的形成过程. 本文对此进行了研究, 观察到了贝氏体层错的产生过 程, 为澄清贝氏体的相变机制提供了重要的实验依据.

合金成分为 (质量分数) $\mathrm{Cu}-25.9 \mathrm{Zn}-4.0 \mathrm{Al}$, 试样经 $800{ }^{\circ} \mathrm{C}$ 等温 $5 \mathrm{~min}$ 后淬人室温水中, 随 后在中温盐浴中等温不同时间, 使贝氏体分别处于不同的生长阶段, 用 $\mathrm{H} 800$ 型透射电镜观察 贝氏体的组织结构, 加速电压为 $200 \mathrm{kV}$.

图 1 是初生贝氏体的明场像. 经双倾台反复倾转, 始终未发现贝氏体内有层错存在. 不 仅如此, 由图中的等厚条纹还可看出, 该初生贝氏体的宽面上有三维台阶存在, 如前所述, 这

1994-12-29 收稿, 1995-05-15 收修改稿

*国家自然科学基金资助项目 
正是初生贝氏体的结构特征, 表明贝氏体在形成初期不是通过切变而是通过台阶机制生长 的. 图 1 中,贝氏体 $\mathrm{A}$ 的一个宽面上有 4 个台阶, 高度约为 $20 \mathrm{~nm}$. 由于片条 $\mathrm{A}$ 由起始端 向生长前端呈由粗变细状, 故有理由认为, 这些台阶是沿贝氏体片条的长轴方向 (图中粗箭头 方向) 运动的, 当它们都运动至片条的生长端部后, 贝氏体 $\mathrm{A}$ 将变成类似于贝氏体 $\mathrm{B}$ 那样的形 貌.

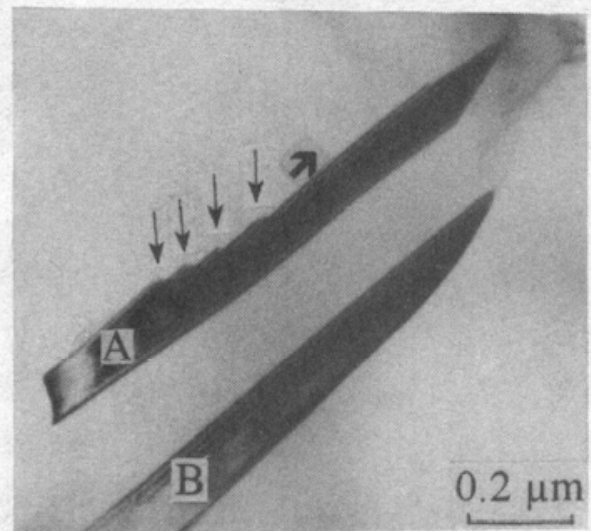

图 1 贝氏体的初生态 $\left(250^{\circ} \mathrm{C}, 360 \mathrm{~s}\right)$

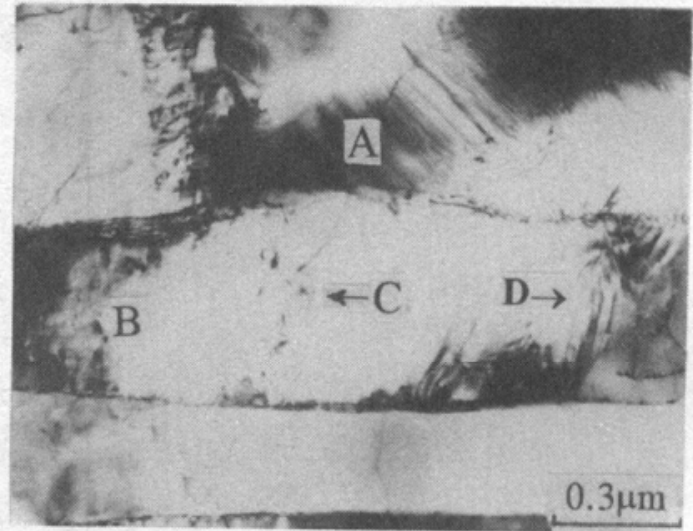

图 2 贝氏体的退化态 $\left(350^{\circ} \mathrm{C}, 1200 \mathrm{~s}\right)$

图 2 是退化态贝氏体的显微结构. 图中也有两个贝氏体片 ( $\mathrm{A}$ 和 $\mathrm{B}$ ), 其中 $\mathrm{A}$ 处于退化的 前期, 其层错基本完整; 贝氏体 $\mathrm{B}$ 中的层错则已全部消失, 内部仅存在无规律的位错线. 在局 部区域位错发生缠结, 呈网状分布, 分别如箭头 C, D 所示.

图 3 显示的是贝氏体层错正处于形成阶段的情形. 图中, 贝氏体片的宽度约为 $0.6 \mu \mathrm{m}$, 长 度大于 $3 \mu \mathrm{m}$, 显然这是生长到一定程度的贝氏体, 而不可能是贝氏体的晶核或刚形成不久的 贝氏体. 由图 3(a) 可以清楚地看出, 贝氏体内有两束层错正由其下部宽面向上扩展, 如箭头 所示. 此外,在未出现层错的部分还有位错线.

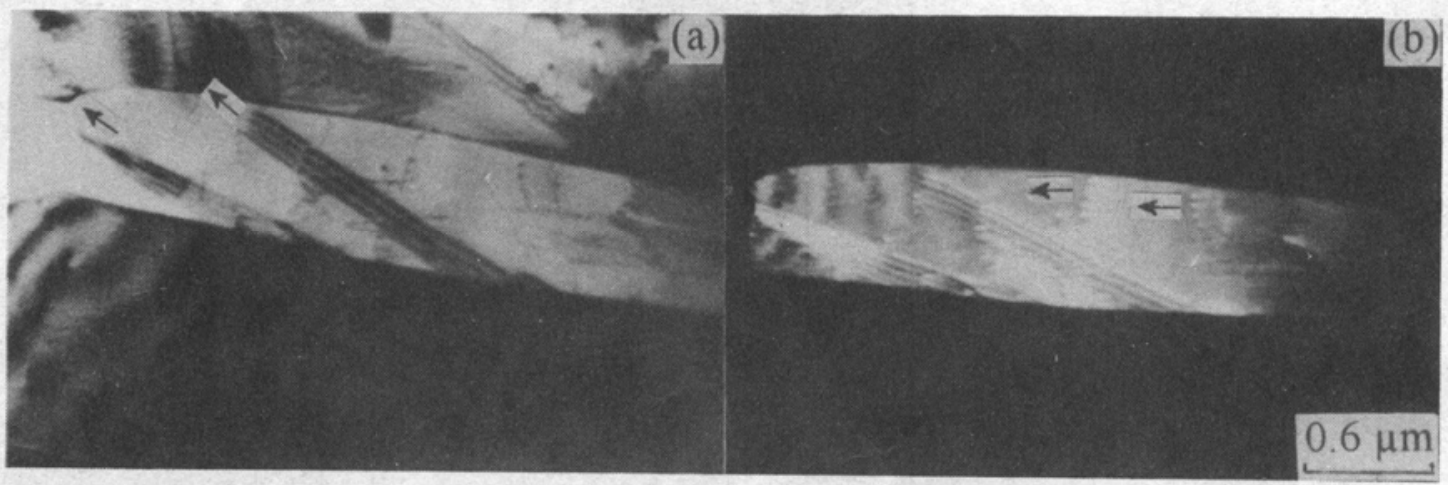

图 3 贝氏体层错的形成 $\left(300^{\circ} \mathrm{C}, 400 \mathrm{~s}\right)$

(a) 明场象, (b) 暗场象

需要说明的是, 刚出现层错的中间态贝氏体与退化阶段的贝氏体是不同的,这可从图 2 及 图 3 中很容易地区分开来: 退化态贝氏体中层错消失部分的界面不再保持平直, 而是向外凸 出, 这是由于长时间等温后, 贝氏体中的溶质原子发生长程扩散造成的; 另外, 退化贝氏体内 部的位错线密度较大, 分布杂乱, 无任何规律性. 相反, 中间态贝氏体的界面平直, 内部位错 
密度很小, 分布亦较有规律, 其运动方向大致与贝氏体片的长轴方向平行, 由贝氏体的起始端 向生长前端推进, 如图 3(b) 中箭头所示.

由此可见, $\mathrm{Cu}-\mathrm{Zn}-\mathrm{Al}$ 合金贝氏体在形成时并不伴随有堆垛层错出现, 这表明贝氏体不是 切变形成的产物. 不仅如此, 初生贝氏体的宽面和端面上还有台阶存在. 大量实验结果表 明, 贝氏体的长大与台阶机制密切相关 ${ }^{[10.12]}$. 层错出现于贝氏体生长的第二阶段即中间态, 其 形成与切变无关. 由于该合金系发生贝氏体相变时体积收缩 (经计算, 相变时体积收缩达 3. $5 \%$ ), 因而产生较大的内部应变, 加之黄铜的层错能很低, 仅为 $0.07 \mathrm{~J} / \mathrm{m}^{2[13]}$, 故认为层错是为了 松弛贝氏体的内部应变而产生的 ${ }^{[11]}$.

\section{参考文献}

1 Clark H M, Wayman C M. Surface relief effects in solid-state phase transformations. In: Phase Transformations, Detroit, 1968. Ohio:ASM, 1970. 59 114

2 Christian J W. Physical Properties of Martensite and Bainite. London: Iron and Steel Institute, 1965. 1

3 Lieberman D S. Crystal geometry and mechanisms of phase transformations in crystalline solids. In: Phase Transformations, Detroit, 1968. Ohio:ASM, 1970, 1 58

4 Flewitt P E J, Towner J M. Internal markings in copper/zinc bainite. Acta Metall, 1966, 14:1013 1015

5 Hornbogen E, Warlimont H. Die bainitische umwandlung des $\beta$-messings. Acta Metall, 1967, 22: 943 951

6 Wu M H, Perkins J, Wayman C M. Long range order, antiphase domain structures, and the formation mechanism of $\alpha_{1}$ ("bainite") plates in a Cu-Zn-Al alloy. Acta Metall, 1989, 37: $1821 \sim 1837$

7 韩 明, 陈复民, 陈金铭等. Cu-Zn-Al-Mn 合金的贝氏体中脊利台阶. 金属学报, 1990, 26: A81 85

8 杨延清, 刘东慧, 康涑狂等. $\beta$ 黄铜中贝氏体生长的高温动态研究. 金属学报, 1992, 28: A95 99

9 Chattopadhyay K, Aaronson H I. Interfacial structure and crystallographic studies of transformations in $\beta^{\prime}$ and $\beta$ Cu- $\mathrm{Zn}$ alloys - I. Isothermal formation of $\alpha_{1}$ plates from $\beta^{\prime}$. Acta Metall, 1986, 34: 695 711

10 Fang Hong-sheng, Li Chun-ming. Study on the transformation mechanism of $\alpha_{1}$ plates in a Cu-Zn-Al alloy. Metall Mater Trans, 1994, 25A:2615 2619

11 李春明, 方鸿生, 郑燕康等. $\mathrm{Cu}-\mathrm{Zn}-\mathrm{Al}$ 合金贝氏体的三个生长阶段及其精细结构研究. 电子显微学报, 1995, 14:118 122

12 李春明,方鸿生. Cu-Zn-Al 合金中贝氏体的纳米级生长台阶。科学通报, 1994, 39(11): 980 982

13 Smallman R E. Dislocations in Crystals, Modern Physical Metallurgy, 4th ed. London: Butterworths, 1985. 227 